\title{
DIRICHLET $L$-FUNCTIONS AND PRIMITIVE CHARACTERS
}

\author{
TOM M. APOSTOL
}

\begin{abstract}
It is well known that a Dirichlet $L$-function $L(s, \chi)$ has a functional equation if the character $\chi$ is primitive. This note proves the converse result. That is, if $L(s, \chi)$ satisfies the usual functional equation then $\chi$ is primitive.
\end{abstract}

1. Introduction. For a positive integer $k$, let $\chi$ be any character modulo $k$, let $L(s, \chi)$ denote the $L$-function defined for $R(s)>1$ by the Dirichlet series

let $G(n, k)$ denote the Gauss sum

$$
L(s, \chi)=\sum_{n=1}^{\infty} \frac{\chi(n)}{n^{s}}
$$

$$
G(n, k)=\sum_{h=1}^{k-1} \chi(h) e^{2 \pi i n h / k},
$$

and let $G(\chi)=G(1, \chi)$. It is well known that if $\chi$ is primitive then $L(s, \chi)$ satisfies the functional equation

(1) $L(1-s, \chi)=(2 \pi)^{-s} \Gamma(s) k^{s-1}\left\{e^{-i s \pi / 2}+\chi(-1) e^{i s \pi / 2}\right\} G(\chi) L(s, \bar{\chi})$.

A recent proof is given in [1]. This paper proves the converse result.

THEOREM 1. If $\chi$ is a character modulo $k$ and if $L(s, \chi)$ satisfies the unctional equation (1), then $\chi$ is a primitive character modulo $k$.

The proof is based on two lemmas, each of which gives a necessary and sufficient condition for a character modulo $k$ to be primitive.

2. Lemmas. The first lemma is a restatement of Theorem 1 in [2].

LeMma 1. A character $\chi$ modulo $k$ is primitive if, and only if,

$$
G(n, \chi)=\bar{\chi}(n) G(\chi)
$$

for every integer $n$.

Received by the editors April 22, 1971.

AMS 1970 subject classifications. Primary 10H10; Secondary 30A20.

Key words and phrases. Dirichlet $L$-functions, primitive characters, functional equations.

(c) American Mathematical Society 1972 
The next lemma involves the function $F(x, s)$ defined for each real $x$ as the analytic continuation of the Dirichlet series

$$
F(x, s)=\sum_{n=1}^{\infty} \frac{e^{2 \pi i n x}}{n^{s}}, \quad R(s)>1 .
$$

This function was used recently in [1] to give a new representation of $L(s, \chi)$ for primitive characters.

LeMma 2. For any character $\chi$ modulo $k$ and any complex $s$, let

Then we have

$$
L^{*}(s, \chi)=\sum_{h=1}^{k-1} \chi(h) F\left(\frac{h}{k}, s\right)
$$

$$
L^{*}(s, \chi)=G(\chi) L(s, \bar{\chi})
$$

for all $s$ if and only if $\chi$ is primitive.

Proof. If $R(s)>1$ we have

$$
L^{*}(s, \chi)=\sum_{h=1}^{k-1} \chi(h) \sum_{n=1}^{\infty} n^{-s} e^{2 \pi i n h / k}=\sum_{n=1}^{\infty} G(n, \chi) n^{-s}
$$

and

$$
G(\chi) L(s, \bar{\chi})=\sum_{n=1}^{\infty} G(\chi) \bar{\chi}(n) n^{-s} .
$$

If (2) holds for all $s$ then it also holds for $R(s)>1$ and the two Dirichlet series in (3) and (4) have the same coefficients. By Lemma 1 it follows that $\chi$ is primitive.

Conversely, if $\chi$ is primitive, Lemma 1 shows that the two functions in (3) and (4) are equal for $R(s)>1$ and hence they must be equal for all $s$.

3. Proof of Theorem 1. We refer to equation (39) in [1] and note that it is valid for every character $\chi$ modulo $k$. This gives us the relation

$$
L(1-s, \chi)=f(s, \chi) L^{*}(s, \chi)
$$

where

$$
f(s, \chi)=(2 \pi)^{-s} \Gamma(s) k^{s-1}\left\{e^{-i s \pi / 2}+\chi(-1) e^{i s \pi / 2}\right\} .
$$

If $L(s, \chi)$ satisfies the functional equation (1) we also have

$$
L(1-s, \chi)=f(s, \chi) G(\chi) L(s, \bar{\chi}) .
$$

From (5) and (6) we find $G(\chi) L(s, \bar{\chi})=L^{*}(s, \chi)$ for all $s$. Therefore, by Lemma $2, \chi$ is primitive. 


\section{REFERENCES}

1. Tom M. Apostol, Dirichlet L-functions and character power sums, J. Number Theory 2 (1970), 223-234. MR 41 \#3412.

2. - Euler's $\varphi$-function and separable Gauss sums, Proc. Amer. Math. Soc. 24 (1970), 482-485. MR 41 \#1661.

Department of Mathematics, California Institute of Technology, Pasadena, California 91109 